\title{
Research
}

\section{Symptom management for medically unexplained symptoms in primary care:}

a qualitative study

\begin{abstract}
\section{Background}

GPs have a central position in the care of patients with medically unexplained symptoms (MUS), but GPs find their care challenging. Currently, little is known about symptom management by GPs in daily practice for patients with MUS.
\end{abstract}

\section{Aim}

This study aimed to describe management strategies used by GPs when confronted with patients with MUS in daily practice.

\section{Design and setting}

Qualitative study in which videos and transcripts of 39 general practice consultations involving patients with MUS in the region of Nijmegen in the Netherlands in 2015 were analysed.

\section{Method}

A thematic analysis of management strategies for MUS used by GPs in real-life consultations was performed.

\section{Results}

The study revealed 105 management strategies in 39 consultations. Nearly half concerned symptom management; the remainder included medication, referrals, additional tests, follow-up consultations, and watchful waiting. Six themes of symptom management strategies emerged from the data: cognitions and emotions, interaction with health professionals, body focus, symptom knowledge, activity level, and external conditions. Advice on symptom management was often non-specific in terms of content, and ambiguous in terms of communication.

\section{Conclusion}

Symptom management is a considerable part of the care of MUS in general practice. GPs might benefit from support in how to promote symptom management to patients with MUS in specific and unambiguous terms.

\section{Keywords}

medically unexplained symptoms (MUS); health communication; qualitative research; primary care; symptom management.

\section{INTRODUCTION}

Medically unexplained symptoms (MUS) are somatic symptoms that cannot be attributed to a clear organic cause after adequate medical examination. ${ }^{1}$ The severity of MUS ranges from mild, self-limiting symptoms to severe, disabling disorders. Depending on the exact definition, the prevalence of MUS is estimated to be $3-20 \%$ of consultations in general practice. ${ }^{2-5}$

Under current guidelines, GPs have a central position in the care of patients with MUS. ${ }^{6-8}$ Though GPs agree that patients with MUS should be managed in primary care, many perceive these patients to be difficult, ${ }^{9-11}$ with a dilemma over whether or not to pursue further diagnostic evaluations. GPs often feel pressurised to undertake or refer for further diagnostic testing, but studies show that it may be the GPs themselves and not the patients who initiate these strategies. ${ }^{12}$ Pursuing further diagnostic investigations might have more negative than positive effects on symptoms and associated cognitions and emotions. ${ }^{13,14}$

GPs could also focus on symptom management, and provide advice about what patients can do themselves about their symptoms. A recent meta-analysis showed that self-help for MUS was associated with a significant reduction in symptom severity and improvement of quality of life. ${ }^{15}$ In accordance with these findings, current guidelines recommend GPs to encourage activating behaviour and to give practical and positive advice that patients can apply straight away. ${ }^{16}$

However, it is largely unknown what GPs do in daily practice when confronted with patients with MUS. GPs are trained to diagnose and treat disease, and might not have a large repertoire of symptom management strategies available. Studies on GP approaches to MUS are largely based on indirect accounts of consultations. A recent meta-synthesis of qualitative studies on management of patients with MUS in primary care identified 13 studies, all based on focus groups and interviews. ${ }^{17}$ These studies mainly focused on explanations and relational aspects, and thus lacked information on GPs' actions with regard to stimulating symptom management. The few observational studies available focused on the topic of somatic interventions. ${ }^{12,18}$ One recent study explored a broader range of management strategies, and reported that GPs provide advice, mainly with regard to lifestyle, diet, and exercise. However, analyses were performed on routinely
J Gol, MD, psychiatrist; T Terpstra, MD, medical doctor; J Rosmalen, PhD, professor, Interdisciplinary Center Psychopathology and Emotion Regulation, University Medical Center Groningen, University of Groningen, Groningen, the Netherlands. P Lucassen, MD, PhD, GP and senior research fellow; J Houwen, MD, GP, Radboud Institute for Health Sciences, Department of Primary and Community Care, Radboud University Medical Center, Nijmegen, the Netherlands. S van Dulmen, PhD, professor, Netherlands Institute for Health Services Research (NIVEL), Utrecht, the Netherlands, and Faculty of Health and Social Sciences, University of South-Eastern Norway, Drammen, Norway. TC Olde Hartman, MD, PhD, GP and senior research fellow, Donders Institute for Brain Cognition and Behaviour, Department of Primary and Community Care, Radboud University
Medical Center, Nijmegen, the Netherlands. Address for correspondence

Janna Gol, Interdisciplinary Center Psychopathology and Emotion Regulation, University Medical Center Groningen, University of Groningen, PO Box 30.001, 9700 RB Groningen the Netherlands.

Email: j.m.goldumcg.nl

Submitted: 18 July 2018; Editor's response: 8 August 2018; final acceptance: 12 November 2018.

\section{CBritish Journal of General Practice}

This is the full-length article (published online 12 Mar 2019) of an abridged version published in print. Cite this version as: Br J Gen Pract 2019; DOI: https://doi.org/10.3399/bjgp19X701849 


\section{How this fits in}

This study explores strategies GPs use in daily general practice when they encounter patients with medically unexplained symptoms (MUS), with a focus on symptom management. It provides an overview of management strategies, which can inspire further studies and training programmes for GPs, with the ultimate aim of improving care for patients with MUS in general practice.

collected health care data extracted from medical records of patients participating in a trial. ${ }^{19}$ The study noted that GPs varied in the way that they recorded what happened during the consultation, so it can be questioned whether this study provided a complete picture.

Knowledge about symptom management strategies that GPs use in daily practice is thus largely lacking. Such knowledge is essential to inform the development of new interventions and training programmes. Therefore, the aim of this study is to describe symptom management by GPs, using video recordings of real-life MUS consultations.

\section{METHOD}

\section{Sample}

Video recorded consultations and verbatim transcripts were collected in the course of the Clinical Assessment as Therapy in managing Medically Unexplained Symptoms project (CATMUS). CATMUS aims to develop an effective and acceptable intervention for MUS for GPs, which can be applied as part of a regular consultation. ${ }^{20}$ GPs in the region of Nijmegen in the Netherlands were actively approached for participation by one of the researchers. The study used purposive sampling to ensure that participating GPs varied in terms of age, sex, experience llength of time practising), and geographical location (city versus rural area). Once GPs had agreed to participate, one of the researchers visited the practice to inform the GPs about the study. They were told this study was about communication between GP and patient, with a special focus on MUS.

Data collection took place in 2015. One of the researchers was present for 1 or 2 days in the primary care practices. He invited all patients in the waiting room during that time who were aged $\geq 18$ years and spoke Dutch fluently to participate. Patients were informed that the study was about communication between GPs and patients, without mentioning the special interest in
MUS. In all, 509 patients were eligible, of whom 393 agreed to participate $(77 \%)$. Consultations were video recorded without researchers present in the consulting room.

MUS was defined by the participating GPs. Immediately after the consultation, the GP answered the following question on a three-point scale (MUS, partial MUS, MES [medically explained symptoms]): 'Do you think this patient has MUS considering his/her physical symptom presentation? The analysis was based on patients who had been classified as MUS by their GP. the partial MUS group was excluded. This ensured that GPs dealing with what they perceived to be MUS were observed. Using GPs' opinion to identify an MUS consultation has also been used in previous research. ${ }^{21,22}$ To check the initial MUS diagnosis, 3 months after the consultation GPs were asked whether an underlying somatic disease was found during the follow up; this only occurred once. All MUS consultations from the CATMUS sample were analysed; 39 consultations with 39 unique patients and 18 GPs. Participant characteristics can be found in Table 1 . Based on previous qualitative studies, this sample was expected to be sufficient for an in-depth description of provided symptom management. ${ }^{12,23}$

\section{Analysis}

This study is a thematic analysis of management strategies for MUS used by GPs in real-life consultations. The method of thematic analysis was chosen as a first step to gain a comprehensive overview of key management strategies for future in-depth studies. ${ }^{24}$ Observation and coding of all consultations was independently performed by a male general medical doctor and a female psychiatrist specialising in somatic symptom disorders and with a degree in social anthropology. During the analytical process, they frequently discussed coding, categories, and themes with senior researchers; a female medical biologist and psychologist specialising in MUS research, and two male GPs experienced in performing qualitative research, to address possible biases.

The analysis followed the following steps. First, a management strategy was defined as every effort proposed by the GP to be undertaken by the MUS patient in the nearby future to promote his or her physical and or emotional wellbeing, in and outside the medical realm'. Based on the authors experience as clinicians, and the literature, ${ }^{25,26}$ the following categories of management strategies 


\section{Table 1. Characteristics of participants}

\begin{tabular}{lcc} 
& GPs & Patients with MUS \\
\hline $\begin{array}{l}\text { Age, mean years } \\
\text { (min-max) }\end{array}$ & $46(31-69)$ & $53(19-88)$ \\
\hline $\begin{array}{l}\text { Sex, } n(\%) \\
\text { Female }\end{array}$ & $9(50)$ & $28(72)$ \\
Male & $9(50)$ & $11(28)$ \\
\hline $\begin{array}{l}\text { Working experience, } \\
\text { mean years (min-max)) }\end{array}$ & $15(2-43)$ & - \\
\hline $\begin{array}{l}\text { Geographical location } \\
\text { of practice, } n(\%)\end{array}$ & & - \\
$\quad$ City & & \\
Rural & $8(44)$ & - \\
\hline Level of education, $n(\%)$ & $10(56)$ & - \\
$\quad$ Low & & $5(13)$ \\
Medium & - & $22(56)$ \\
$\quad$ High & - & $12(31)$ \\
\hline $\begin{array}{l}\text { Voluntary or paid } \\
\text { work, } n(\%)\end{array} \quad-$ & \\
$\quad$ Yes & - & $17(44)$ \\
No & - & $22(56)$ \\
\hline
\end{tabular}

a Education level was classified as low (primary education), medium (secondary education), and high (pre-university and university. $\max =$ maximum. $\min =$ minimum. MUS = medically unexplained symptoms. were expected: additional tests, referrals, medication, watchful waiting, and symptom management. Symptom management was defined as advice about what patients can do themselves to reduce the impact, severity, or frequency of their symptoms, outside the medical realm'. Second, a detailed line-by-line analysis was applied to codeobserved management strategies as part of one of these pre-defined categories (closed codingl, or as part of a newly emerging category (open coding). This was performed independently by two researchers for every consultation. After watching, reading, and coding a consultation, discussion took place between the two researchers, in an iterative process of seeking agreement, adjusting codes, and recoding consultations. Data from the verbatim transcripts were organised in Atlas.ti 7. Saturation in terms of management strategy categories was reached after analysing nine consultations, while no new themes of symptom management strategies were found after analysing 24 consultations. Third, once all management strategies had been extracted from the transcripts, a thematic analysis was performed on the category of symptom management using One Sheet Of Paper analysis (OSOP). OSOP analysis is a way of visually rearranging the extracted data in order to define themes. ${ }^{27}$ Summaries of all symptom management quotations were written on a single sheet of paper, rearranging them by looking at similarities and differences. As a result, themes emerged, describing the variety of symptom management by GPs. As the summaries also included the contextual embedding of symptom management, this yielded insights into the communication aspects related to how symptom management is promoted by GPs. For the results section, quotations that illustrated the essence of the themes were selected. To ensure intersubjective reproducibility and comprehensibility, the results were presented to and discussed frequently with senior researchers throughout the process of analysis.

\section{RESULTS}

In 39 consultations, 105 management strategies were identified; nearly half concerned symptom management. All but one consultation contained at least one management strategy, with a maximum of six strategies per consultation. The closed coding strategy identified all pre-defined management strategies: additional tests, referrals, medication, watchful waiting, and symptom management.

\section{Symptom management}

The in-depth analysis of symptom management resulted in the identification of six themes: cognitions and emotions, interaction with healthcare professionals, body focus, symptom knowledge, activity level, and external conditions.

Before discussing these themes in detail, three general observations are worth mentioning. First, a great variety of advised symptom management strategies was observed. Second, communication about symptom management strategies was often non-specific instead of practical. Thus, it remained unclear how suggested symptom management strategies should be carried out by the patient. Third, symptom management strategies were often presented in an ambiguous fashion. In these instances, GPs suggested a certain symptom management strategy while at the same time raising doubts about its potential effectiveness, for example, adding that it is only an idea', or 'it is unknown whether it will actually help or not'.

Cognitions and emotions. Symptom management strategies focusing on cognitions and emotions were prominent, with an emphasis on cognitions. These were most often initiated by the GP, and were often communicated as an order, for example, 'concerning your memory, don't be so worried. These orders were brief phrases, 
and GPs rarely provided specific practical instructions to patients. For instance, patients were advised to accept their complaints: 'So, I reckon we should just accept it, leave it be ...., but they were not instructed how to do this. The same absence of specific practical instructions was observed in advice on setting priorities: 'Yeah, well, you constantly have to weigh in mind; with what do I gain, with what do I lose, what is still achievable? and, in another consultation: 'You are going to set priorities, what is most important, and on what do I want to spend energy? Apart from one GP, emotion-focused advice was hardly observed, and virtually always concerned fear

Interaction with healthcare professionals. In this theme, patients were advised and/or instructed on how to deal with healthcare professionals. In contrast to the theme of cognitions and emotions, symptom management advice related to interactions with health professionals was communicated in an open two-way conversation in which GPs regularly discussed their own position. In general, advice within this theme was clear and comprehensive. For example, when a patient asked whether she should try haptonomy as well as psychological treatment, she was told: 'Because you're that sensitive, make sure there is one person in charge of your treatment, so I would advise to attend only the psychologist now. The contextual embedding of this kind of symptom management was mainly support: GPs explained their role to the patient and their willingness to help, for example:

'The moment you realise it doesn't feel right, that you're losing it, you can always come. Then we can try to improve or adjust. (GP)

I mean, you say your past means that you get worried sooner, so it's logical that you're here. And, it's part of my job as a GP to reassure people who are worried. Justified or not. It's called guidance. So, you must come whenever you want to. Not a problem.' (GP)

Body focus. This theme included practical symptom management advice about posture, breathing, relaxation, home remedies, and nutrition. These symptom management strategies mostly resulted from a dialogue between the GP and the patient, or from a direct question of the patient. For instance, one patient asked: 'Can I also treat it with, well I don't know if it works, tiger balm? Whereupon the GP replied with: 'Yes, that's fine.' Suggested symptom management strategies in this category were often short and non-specific, for example, keep a relaxing posture at work, and a good posture with sports', and they were sometimes communicated in an ambiguous manner. One GP replied to a patient's question with: 'Massage, that's good, feels pleasant. We actually never know whether it fastens [the healing] or...' Another GP advised stopping drinking alcohol during the week, while approving it at the weekend, even though he mentioned this might negatively influence stopping it during the week:

GP: 'Four glasses a day is a lot, so it's a good idea to cut down."

Patient (P): 'Yes, I just did, and now I've only got it for the weekend. I do drink then. I want to, because otherwise ...'

GP: 'Should be okay.

P: 'Yes, that's what I think, see how it goes. GP: 'I can imagine that it might make it harder during the week. But it's a matter of trying.

P: 'Right, it is. I'll just try and see how it goes."

Symptom knowledge. GPs used two ways to increase their patients' knowledge about their symptoms. First, GPs suggested patients register their symptoms in a diary. Examples included a patient with fibromyalgia who was advised to keep a pain diary, and a patient with symptoms of fainting who was advised to register blood pressure.

The GPs explained to their patients that the aim of this symptom management strategy was to gain further insight into their symptoms. However, in none of the consultations did it become fully clear what the GP aimed to do with these registered data:

GP: We can do two things. We could stop the hydrochlorothiazide. Then, if your blood pressure drops, you don't feel dizzy; but that means that if your blood pressure is high, your blood vessels will suffer in the long run. That's one. Another option is that we, that you keep a logbook for 2 weeks, write down how you were every day, what your blood pressure was, how you felt, and that you take your blood pressure 3 times a day, and if you don't feel well, you take your blood pressure again if you can.

P: 'I usually do that if I don't feel well."

GP: 'Exactly. But then we'll, well, we'll see what happens. Because if you're very sensitive to what's going on around you and 
you're affected by it, then this might make your blood pressure drop, and then we might need to do something about it.'

Second, GPs provided leaflets with information about symptoms. Presenting a leaflet occurred in one consultation as follows: '... giving you a leaflet ... it might provide tools, but, well, it is not going to help you for this episode, I think, by which the GP raised doubts about the potential effectiveness of the leaflet. Strategies related to symptom knowledge were always initiated by the GP.

Activity level. The essence of this theme is that patients are advised to change their activity level. Of note is the fact that all GPs in this sample advised patients to stay at the current activity level or to do less.

Patients were instructed not to do too much during the day and to maintain a daily rhythm with sufficient rest. None of the GPs suggested increasing activity levels. When GPs confirmed already performed activities of the patients, they did so in specific and unambiguous terms.

In contrast, when they provided advice on activity levels, it was non-specific, for example: 'So, in your holiday you can make sure you are at rest and you are able to recuperate.

External conditions. This theme contains symptom management strategies focused on adjustments at work or at home. Such adjustments were presented as a solution for a proposed perpetuating factor. This solution was communicated quite ambiguously in a case in which the GP recommended a stair lift, while repeatedly commenting on the high costs of it:

P: Walking upstairs is difficult. Once I'm up there, it's like I'm totally done in, pffff. Everything's knotted up, and I can't get it undone. It's, it's hell. Like you're in hell. And before the worst of the pain's gone, and I don't know how else to do it, whether to count to three and have a rest, and then carry on, or just get on with it, it doesn't make any difference.

GP: And, are you ready to give up the stairs?' P: 'I am. I wouldn't mind a stair lift. The stairs take all my energy.'

Patient's husband (PH): 'Yes, no, I'm up for it. If we must, we must.

P:'Yes, but it's expensive. Costs a lot of money, eh?

GP: 'Costs a lot of money. But haven't you, because your house is quite big, haven't you got a downstairs bedroom?'
PH: 'Yes, there is one. We can make one. It's an office at the moment, but we can turn it into a bedroom, but the shower's still upstairs. You'd have to install a shower downstairs.

GP: 'Yes, that's expensive too.

\section{DISCUSSION}

\section{Summary}

This study showed that symptom management is a considerable part of GP care for MUS, as it comprised nearly half of all observed management strategies. Besides symptom management, GPs promote additional tests, referrals, medication, follow-up consultations, and watchful waiting. An in-depth analysis of symptom management strategies identified six themes: cognitions and emotions, interaction with healthcare professionals, body focus, symptom knowledge, activity level, and external conditions. Advice on symptom management was often non-specific and communicated in an ambiguous manner.

\section{Strengths and limitations}

To the best of the authors' knowledge, this study is the first to address advised management strategies for MUS by observing and analysing what GPs do in daily practice. Focus groups and interview studies give insight into experiences and opinions on dealing with MUS, ${ }^{25,26,28}$ whereas this research method provided a picture of GPS acting on MUS in daily practice. One recent study also analysed management strategies in daily practice, but was based on routinely collected healthcare data extracted from medical records of patients participating in a trial of treatment of MUS. ${ }^{19}$ Such a trial likely involves a specific group of GPs interested in MUS, and the routine coding of management strategies varied among GPs and was not as detailed as in this study. Another strength of the current study is that the GPs themselves decided whether it was an MUS consultation or not, ensuring that the findings were based on what GPs did when confronted with patients they considered to be patients with MUS. To check the initial MUS diagnosis, GPs were asked 3 months after the consultation whether an underlying somatic disease was found during the follow-up; this only occurred once. However, even if a GP's initial assessment is incorrect, it will still determine the management strategies proposed in that consultation. A major advantage of relying on GPs' interpretation is that it takes into account the heterogeneity between GPs in what they consider to be MUS.

The study also has limitations. Apart 
from age and sex, no data were available on the 116 patients who did not agree to participate. Therefore, the risk of selection bias cannot be completely ruled out. However, the sample of patients varied in terms of sex, age, and type of complaints. Another potential drawback is the risk that communication behaviour was altered because the consultations were video recorded. However, research on video recording reports no significant effects on the behaviour of patients and doctors. ${ }^{29-31}$ Most patients in this study claimed that they had not noticed any change in their doctor's behaviour, while most of these doctors said they experienced no difficulties in ignoring the camera. ${ }^{20} \mathrm{~A}$ further limitation is that the results were based on one consultation, with no information about previous and future consultations. However, the study as a whole included first consultations as well as follow-up consultations, resulting in a comprehensive overview of management strategies for MUS. Nevertheless, it was not possible to analyse how symptom management strategies develop in a care episode involving multiple consultations.

A final limitation is that the entire data collection was done before data analysis. Saturation in terms of management strategy categories and themes of symptom management was reached, but this does not mean that all possible symptom management strategies advised by GPs were covered. Though the population was centred in only one part of the Netherlands, the participating GPs did not have a special interest in MUS and varied in terms of age, sex, experience, and geographical location (city versus rural area).

\section{Comparison with existing literature}

A recent review has indicated that a broad array of self-help interventions is effective for the alleviation of symptoms and improvement of functioning in patients with MUS. ${ }^{15}$ This review included structured self-help treatments that are not routinely available for most GPs. However, it emphasises the potential of symptom management, in which patients have to act themselves on their symptoms. Participating GPs seem to endorse this, as nearly half of all observed management strategies concerned symptom management. From the current study, conclusions on whether the various symptom management strategies are associated with better or worse outcomes for patients cannot be drawn. However, several of these strategies are essential components of current treatments, such as changing cognitions and emotions in cognitive behavioural therapy, changing activity levels in graded exercise therapy, increasing symptom knowledge in psychoeducation, body focus in brief multimodal psychosomatic therapy and physiotherapy, and changing external conditions in occupational therapy. ${ }^{32,33}$ Thus, indirect evidence suggests that the various symptom management strategies identified in this study might be effective. However, their potential is not used to the full because observed symptom management was often non-specific instead of practical, and communicated in an ambiguous manner. This fits with the observation that patients with MUS identified the absence of a specific management plan as one of the problems experienced in the consultation, with GPs unclear about what patients should do after leaving the consultation room. ${ }^{34}$ That the potential of symptom management is not fully used seems in line with GPs finding MUS difficult to manage, $, 10,35-39$ and evidence suggesting that GPs think effective treatments are lacking. ${ }^{9}$ In addition, relevant information regarding symptom management, such as information on characteristics of MUS patients who are most likely to respond, is lacking.

Though many GPs are convinced that psychological factors play a role in MUS, ${ }^{28,40}$ the management strategies GPs used in this study do not support this conviction. The majority of all management strategies, as well as many observed symptom management strategies, were somatically oriented. This reflects the dominant medical model under which GPs are trained. In accordance with previous literature, patients seldom request a somatic intervention. ${ }^{12}$ In addition, referrals for psychological interventions were not observed in this study, even though guidelines recommend them. ${ }^{32}$ Discussions of psychological elements, such as perpetuating factors and how to change these, were rarely observed. These findings are in accordance with a recent study based on routinely collected healthcare data extracted from medical records of patients participating in a trial. ${ }^{19}$ In that study, the least frequently used strategies were exploration of perpetuating factors and referral to mental health care. ${ }^{19}$

\section{Implications for research and practice}

For future research, it would be interesting to study the process leading to specific management strategies. One question would be whether the number of previous consultations influences the type of management strategies and the way in 
which they are communicated to patients. Previous studies suggest that management strategies might differ according to the phase in the care episode. ${ }^{19,25,40}$ It would also be interesting to study whether the choice of management strategy is coupled to specific explanatory models used by the GP. ${ }^{26,41,42}$ Future studies could also focus on the adoption of strategies, and whether this is influenced by the specificity and clarity with which they are communicated. How strategies are perceived by the patient might have a large impact on their potential effects. The effectiveness of management strategies deserves further study, also in relation to diversity, severity, or chronicity of complaints, or other patient characteristics, such as sex or age. Studies in which explanations, management strategies, and their effectiveness are combined, and studies about patients' evaluations of management strategies, have the potential to improve the care for MUS in general practice.

In clinical practice, this study suggests that GPs should provide patients with a specific management plan that is clear about what patients can do after leaving the consultation room. Such a plan can be based on a discussion between GP and patient about symptom management strategies that might be helpful. Though there are no data on whether these specific strategies are associated with better or worse outcomes, circumstantial evidence suggests that they are a good starting point to collaboratively build patient-centred interventions.

\section{Funding}

This study is supported by ZonMw lfunding number 839110010).

\section{Ethical approval}

The research ethics committee of the Radboud University Nijmegen Medical Center approved the study (2015-1566). Written informed consent was obtained from all participating patients; patients were able to withdraw their consent at any time.

\section{Provenance}

Freely submitted; externally peer reviewed.

\section{Competing interests}

The authors have declared no competing interests.

\section{Contributors}

Janna Gol and Tom Terpstra contributed equally to the authorship of this paper.

\section{Discuss this article}

Contribute and read comments about this article: bjgp.org/letters 


\section{REFERENCES}

1. van der Weijden $T$, van Velsen M, Dinant GJ, et al. Unexplained complaints in general practice: prevalence, patients' expectations, and professionals' testordering behavior. Med Decis Making 2003; 23(3): 226-231.

2. Peveler R, Kilkenny L, Kinmonth AL. Medically unexplained physical symptoms in primary care: a comparison of self-report screening questionnaires and clinical opinion. J Psychosom Res 1997; 42(3): 245-252

3. Aamland A, Malterud K, Werner EL. Patients with persistent medically unexplained physical symptoms: a descriptive study from Norwegian general practice. BMC Fam Pract 2014; 15: 107

4. Verhaak PFM, Meijer Sa, Visser AP, Wolters G. Persistent presentation of medically unexplained symptoms in general practice. Fam Pract 2006; 23(4): 414-420.

5. Swanson LM, Hamilton JC, Feldman MD. Physician-based estimates of medically unexplained symptoms: A comparison of four case definitions. Fam Pract 2010; 27(5): 487-493.

6. Olde Hartman TC, Rosendal M, Aamland A, et al. What do guidelines and systematic reviews tell us about the management of medically unexplained symptoms in primary care? BJGP Open 2017; DOI: https://doi.org/10.3399/ bjgpopen17X101061.

7. Olde Hartman TC, Woutersen-Koch H, Van der Horst HE. Medically unexplained symptoms: evidence, guidelines, and beyond. Br J Gen Pract 2013; DOI: https:// doi.org/10.3399/bjgp13X675241.

8. van der Feltz-Cornelis CM, Hoedeman R, Keuter EJ, Swinkels JA. Presentation of the multidisciplinary guideline medically unexplained physical symptoms (MUPS) and somatoform disorder in the Netherlands: disease management according to risk profiles. J Psychosom Res 2012; 72(2): 168-169.

9. Reid S, Whooley D, Crayford T, Hotopf M. Medically unexplained symptoms GPs' attitudes towards their cause and management. Fam Pract 2001; 18(5): 519-523.

10. Hartz AJ, Noyes R, Bentler SE, et al. Unexplained symptoms in primary care: perspectives of doctors and patients. Gen Hosp Psychiatry 2000; 22(3): 144-152.

11. Johansen ML, Risor MB. What is the problem with medically unexplained symptoms for GPs? A meta-synthesis of qualitative studies. Patient Educ Couns 2017; 100(4): 647-654

12. Ring A, Dowrick C, Humphris G, Salmon P. Do patients with unexplained physical symptoms pressurise general practitioners for somatic treatment? A qualitative study. BMJ 2004; 328(7447): 1057-1060.

13. Kouyanou K, Pither CE, Rabe-Hesketh S, Wessely S. A comparative study of iatrogenesis, medication abuse, and psychiatric morbidity in chronic pain patients with and without medically explained symptoms. Pain 1998; 76(3): 417-426.

14. Rolfe A, Burton C. Reassurance after diagnostic testing with a low pretest probability of serious disease: systematic review and meta-analysis. JAMA Intern Med 2013; 173(6): 407-416.

15. van Gils A, Schoevers RA, Bonvanie IJ, et al. Self-help for medically unexplained symptoms: a systematic review and meta-analysis. Psychosom Med 2016; 78(6): 728-739.

16. Heijmans M, Olde Hartman TC, van Weel-Baumgarten E, et al. Experts opinions on the management of medically unexplained symptoms in primary care. a qualitative analysis of narrative reviews and scientific editorials. Fam Pract 2011; 28(4): 444-455

17. Johansen ML, Risor MB. What is the problem with medically unexplained symptoms for GPs? A meta-synthesis of qualitative studies. Patient Educ Couns 2017; 100(4): 647-654.

18. Salmon $P$, Humphris $G M$, Ring $A$, et al. Why do primary care physicians propose medical care to patients with medically unexplained symptoms? A new method of sequence analysis to test theories of patient pressure. Psychosom Med 2006; 68(4): 570-577.

19. Sitnikova K, Pret-Oskam R, Dijkstra-Kersten SMA, et al. Management of patients with persistent medically unexplained symptoms: a descriptive study. BMC Fam Pract 2018; 19(1): 88

20. Houwen J, Lucassen PLBJ, Stappers HW, et al. Medically unexplained symptoms: the person, the symptoms and the dialogue. Fam Pract 2017; 34(2): 245-251.
21. Ring A, Dowrick CF, Humphris GM, et al. The somatising effect of clinical consultation: what patients and doctors say and do not say when patients present medically unexplained physical symptoms. Soc Sci Med 2005; 61(7): 1505-1515.

22. Salmon P, Ring A, Humphris GM, et al. Primary care consultations about medically unexplained symptoms: how do patients indicate what they want? J Gen Intern Med 2009: 24(4): 450-456.

23. Olde Hartman TC, van Rijswijk E, van Dulmen S, et al. How patients and family physicians communicate about persistent medically unexplained symptoms. A qualitative study of video-recorded consultations. Patient Educ Couns 2013; 90(3): 354-360.

24. Kelly M. The role of theory in qualitative health research. Fam Pract 2010; 27(3): 285-290.

25. Hansen HS, Rosendal M, Fink P, Risor MB. The general practitioner's consultation approaches to medically unexplained symptoms: A qualitative study. ISRN Family Med 2012; DOI: 10.5402/2013/541604. eCollection 2013.

26. Olde Hartman TC, Hassink-Franke LJ, Lucassen PL, et al. Explanation and relations. How do general practitioners deal with patients with persistent medically unexplained symptoms: a focus group study. BMC Fam Pract 2009; 10: 68 .

27. Ziebland S, McPherson A. Making sense of qualitative data analysis: An introduction with illustrations from DIPEx (personal experiences of health and illness). Med Educ 2006; 40(5): 405-414.

28. Wileman L, May C, Chew-Graham CA. Medically unexplained symptoms and the problem of power in the primary care consultation: a qualitative study. Fam Pract 2002; 19(2): 178-182

29. Fossum B, Arborelius E. Patient-centred communication: Videotaped consultations. Patient Educ Couns 2004; 54(2): 163-169.

30. Coleman T. Using video-recorded consultations for research in primary care: advantages and limitations. Fam Pract 2000; 17(5): 422-427.

31. Arborelius E, Timpka T. In what way may videotapes be used to get significant information about the patient-physician relationship? Med Teach 1990; 12(2): 197-208.

32. van Dessel N, den Boeft M, van der Wouden JC, et al. Non-pharmacological interventions for somatoform disorders and medically unexplained physical symptoms (MUPS) in adults. Cochrane Database Syst Rev 2014: (11): CD011142.

33. Henningsen P, Zipfel S, Sattel H, Creed F. Management of functional somatic syndromes and bodily distress. Psychother Psychosom 2018; 87(1): 12-31.

34. Houwen J, Lucassen PL, Stappers HW, et al. Improving GP communication in consultations on medically unexplained symptoms: a qualitative interview study with patients in primary care. Br J Gen Pract 2017; DOI: https://doi.org/10.3399/ bjgp17X692537.

35. Garcia-Campayo J, Sanz-Carrillo C, Yoldi-Elcid A, et al. Management of somatisers in primary care: are family doctors motivated? Aust N Z J Psychiatry 1998; 32(4): 528-533.

36. Steinmetz D, Tabenkin H. The 'difficult patient' as perceived by family physicians. Fam Pract 2001; 18(5): 495-500

37. McDonald PS, O'Dowd TC. The heartsink patient: a preliminary study. Fam Pract 1991; 8(2): 112-116.

38. Hahn SR, Thompson KS, Wills TA, et al. The difficult doctor-patient relationship: somatization, personality and psychopathology. J Clin Epidemiol 1994; 47(6): 647-657.

39. Lin EH, Katon W, Von Korff M, et al. Frustrating patients: physician and patient perspectives among distressed high users of medical services. J Gen Intern Med 1991; 6(3): 241-246

40. Woivalin T, Krantz G, Mantyranta T, Ringsberg KC. Medically unexplained symptoms: Perceptions of physicians in primary health care. Fam Pract 2004; 21(2): 199-203.

41. Burton $C$, Lucassen $P$, Aamland $A$, Olde Hartman T. Explaining symptoms after negative tests: towards a rational explanation. J R Soc Med 2015; 108(3): 84-88.

42. van Ravenzwaaij J, Olde Hartman T, van Ravesteijn $\mathrm{H}$, et al. Explanatory models of medically unexplained symptoms: a qualitative analysis of the literature. Ment Health Fam Med 2010; 7(4): 223-231. 